\title{
Paciente oncológico con incapacidad laboral absoluta: características epidemiológicas, supervivencia y seguimiento de su incapacidad laboral
}

\section{Cancer patients with absolut permanent incapacity: epidemiology, survival and monitoring work disability}

\author{
Raúl Jesús Regal Ramos \\ Recibido: 15-03-11 \\ Aceptado: 01-06-11

\section{Correspondencia} \\ Raúl Jesús Regal Ramos \\ Dirección Provincial del Instituto Nacional de la Seguridad Social. \\ c/ López de Hoyos, 169 \\ 28002 Madrid. España. \\ Tfno: 915907432 \\ E-mail: raul-jesus.regal@inss.seg-social.es
}

Dirección Provincial del Instituto Nacional de la Seguridad Social. Madrid. España.

\section{Resumen}

Cada año tanto la incidencia como la supervivencia del cáncer aumentan en España y, paralelamente el gasto económico que supone las Invalidez Permanente Absoluta (IPA) por estas patologías.

Objetivo: Conocer las características epidemiológicas de los pacientes oncológicos con IPA y qué variables se asocian a una supervivencia mayor a 5 años, con la finalidad de establecer unos criterios de revisión de la capacidad laboral de estos pacientes.

Metodo: Se analizaron los pacientes valorados en la Unidad Médica de la Dirección Provincial del Instituto Nacional de la Seguridad Social de Madrid en el año 2005 menores de 55 años, con el diagnóstico de patología oncológica y cuya calificación final fue la de IPA. Se analizaron las siguientes variables: edad, sexo, estado civil, profesión, régimen de afiliación a la Seguridad Social, tipo de cáncer (diferenciando si estaba extendido y/o había tenido recidivas), la presencia de otras patologías asociadas, la presencia de otras limitaciones constitutivas de IPA y por último la supervivencia a los 5 años. De los pacientes que seguían vivos en Noviembre del 2010 se estudió la relación de las variables con la supervivencia y la evolución de su grado de invalidez.

Resultados: El total de pacientes estudiados fueron 212. Los tumores más frecuentes en la mujer fueron mama y pulmón, y en el hombre pulmón y colon-recto. La supervivencia a 5 años fue del $30 \%$ observándose que disminuye con la edad, en función del tipo de tumor, ante la presencia de metástasis y / o recidivas y ante la presencia de comorbilidad. Los tumores que más muertes causan son pulmón en hombres y mama en mujeres. En cuanto al seguimiento de la invalidez, el 54 \% (35 de 64) de los supervivientes ni habían sido revisados ni tenían secuelas u otras patologías constitutivas IPA.

Conclusion: La medicina evaluadora debe adaptarse a los cambios en la evolución de estos pacientes y debe hacerlo actualizando la formación oncológica y aumentando las revisiones. Estas revisiones podrán establecerse en el momento de la calificación de la invalidez o a posteriori, tras seleccionar aquellos casos que, con una supervivencia y edad razonables, no presenten otras limitaciones constitutivas de IPA.

Med Segur Trab (Internet) 2011; 57 (223) 121-133

Palabras claves: Cáncer. Incapacidad. Neoplasia. Pronóstico. 


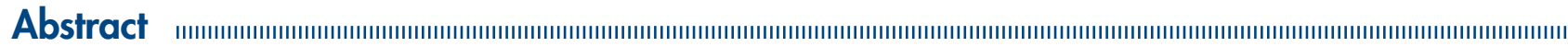

Each year the incidence of cancer in Spain increases, as well as the survival, and at the same time the economic cost due to Absolute Permanent Disability (APD) caused by these diseases.

Objective: Know the epidemiological characteristics of cancer patients with APD and what variables are associated to survival beyond 5 years, in order to establish criteria for the review of the working capacity of these patients.

Method: We analyzed all patients evaluated in the Medical Unit of The National Institute of Social Security in Madrid in 2005, under 55 years of age, with the diagnosis of neoplasic disease and whose final evaluation was APD. We analyzed the following variables: age, sex, marital status, occupation, type of Social Security affiliation, type of cancer (distinguishing whether it was widespread and / or had had relapses), the presence of other associated diseases, the presence of other conditions which in themselves were constitutive of APD and finally the survival beyond 5 years. Among the patients who were alive in November 2010 we studied the relationship of variables with the survival and evolution of the degree of disability.

Results: The total number of patients studied was 212. The most frequent type of tumors in women were breast and lung cancer and in men lung and colon-rectum. The 5-year survival was $30 \%$.This survival decreases with age, depending on the type of tumor, the presence of metastasis and / or relapses and the presence of comorbidity. Tumors that cause most number of deaths are lung cancer in men and breast cancer in women. Regarding the followup of disability, $54 \%$ (35 of 64) of the survivors had not been reviewed and had no sequels or other conditions constituting APD.

Conclusion: Medical evaluation must be adapted to changes in the evolution of these patients and must do so by updating oncological training and increasing medical reviews. These reviews may be established at the same time as the disability rating or later on, after selecting those cases where with a reasonable survival and age, do not exhibit other limitations constitutive of APD.

Med Segur Trab (Internet) 2011; 57 (223) 121-133

Key words: Cancer. Disability. Neoplasia. Prognosis. 


\section{INTRODUCCIÓN}

Cada año la incidencia del cáncer aumenta en España, pero también disminuye su mortalidad, lo cual refleja los avances en el diagnóstico precoz y el tratamiento. Desde el año 2005, los tumores son la primera causa de muerte en los hombres y se mantienen en un segundo lugar en las mujeres, tras las enfermedades cardiovasculares ${ }^{1}$. La Sociedad Española de Oncología Médica (SEOM) en un documento publicado en su página web en Enero de $2009^{2}$ estimaba, con los resultados del Eurocare $4^{3}$ y el informe sobre "Mortalidad por cáncer y otras causas en España" de 2006, elaborado por el Centro Nacional de Epidemiología del Instituto Carlos III, que uno de cada tres españoles y una de cada cuatro españolas se diagnosticarán de cáncer a lo largo de su vida.

Debido al gran número de muertes y secuelas que provocan en los países desarrollados, cada vez son más los estudios que tienen como objetivo el realizar un análisis socioeconómico de los costes de dichas patologías ${ }^{4-6}$. Los costes sanitarios podemos dividirlos en costes directos, que son los que se refieren directamente al consumo de recursos del sistema de atención sanitaria (atención hospitalaria, hospitalizaciones, atención primaria, fármacos, etc.); y los costes indirectos, los relacionados con la disminución o pérdida de productividad debida a la mortalidad prematura y a la incapacidad laboral atribuible a una patología determinada. Según diversos estudios ${ }^{7-9}$, en las enfermedades neoplásicas, el mayor porcentaje de los costes son de tipo indirecto.

Se define como invalidez a la situación del trabajador que tras estar sometido al tratamiento prescrito presenta reducciones anatómicas graves, susceptibles de determinación objetiva y previsiblemente definitivas, que disminuyen o anulan su capacidad labora. La invalidez se define como absoluta (IPA) cuando inhabilita por completo al trabajador para toda profesión u oficio, suponiendo una pensión vitalicia mensual del $100 \%$ de la Base Reguladora ${ }^{10}$. La resolución que indica el grado de invalidez asocia un plazo de revisión. La revisión puede ser de oficio, si se considera que existe la posibilidad de mejoría, o a instancia del interesado.

Debido al aumento de la incidencia y la supervivencia de estos pacientes, y a que los costes sanitarios que generan son en un gran porcentaje derivados de la incapacidad laboral que conllevan, se propone realizar un estudio que tenga como objetivo describir las características epidemiológicas de los pacientes oncológicos con incapacidad laboral absoluta y las variables que se asocian a una supervivencia mayor a 5 años, con el fin de establecer unos criterios de revisión de la capacidad laboral de estos pacientes.

\section{MÉTODO}

Se realizó un estudio observacional de cohortes retrospectivo. Se recogieron todos los pacientes valorados en la Unidad Médica de la Dirección Provincial del Instituto Nacional de la Seguridad Social de Madrid en el año 2005 menores de 55 años, con el diagnóstico de patología oncológica, que habían agotado el plazo máximo de incapacidad laboral temporal (18 meses en el año 2005) y cuya calificación final fue la de IPA (tabla I). Se seleccionaron pacientes menores de 55 años porque uno de los objetivos de este trabajo era conocer la evolución de la incapacidad laboral de estos pacientes. Por tanto, se trataba pues de una población de pacientes oncológicos de mala evolución dado que tras agotar los 18 meses de incapacidad laboral temporal (IT) han recibido la calificación de IPA.

En todos los casos se analizaron las siguientes variables: edad, sexo, estado civil, profesión, régimen de afiliación a la Seguridad Social, tipo de cáncer (diferenciando si estaba extendido y/o había tenido recidivas), la presencia de otras patologías o secuelas recogidas en el informe médico de invalidez, ya sean no constitutivas de IPA (como conectivopatías o insuficiencia cardiaca, renal, hepática o respiratoria) o constitutivas de IPA (como esquizofrenia, trastorno depresivo mayor recurrente, colostomía permanente, 
traqueostoma permanente o hemiparesia) y por último la supervivencia a los 5 años. De los pacientes que seguían vivos en Noviembre del 2010 se estudió la relación de las variables con la supervivencia y la evolución de su grado de invalidez (calificación actual, revisiones previas,...).

Tabla I. Criterios de inclusión

Edad 18 - 55

Valorados en la Unidad Médica de la Dirección Provincial del Instituto Nacional de la Seguridad Social de Madrid en el 2005

Diagnóstico de patología oncológica

Calificación final fue la de IPA*

* IPA: Invalidez Permanente Absoluta

Fuente primaria de información: Unidad de informática de la Dirección Provincial del Instituto Nacional de la Seguridad Social de Madrid

Para el procesamiento de los datos y los cálculos estadísticos se creó una base de datos con Microsoft Excel.

La evolución de estos pacientes desde el punto de vista médico y laboral se refleja en el "esquema del estudio".

\section{CONSIDERACIONES ÉTICAS}

El presente estudio se ha realizado con la autorización de la Dirección General del Instituto Nacional de la Seguridad Social.

La información perteneciente a los pacientes integrantes del estudio se ha tratado de acuerdo a lo establecido en la Ley Orgánica 15/1999 de protección de datos personales.

\section{RESULTADOS}

El total de pacientes estudiados fueron 212. La tabla II recoge las características epidemiológicas de la muestra inicial y de los pacientes a los 5 años de seguimiento, separando los fallecidos de los que continuaban vivos.

La mediana de edad fue de 49 años con una media 46,9 años y una desviación estándar de 7. La trabajadora más joven tenía 22 años. El $41 \%$ tenían entre 51 y 55 años, el $27 \%$ entre 46 y 50, y el $16 \%$ entre 41 y 45. La franja de edad de menos de 41 años supuso el $14 \%$ de los casos. El $62 \%$ de la muestra eran hombres. El $68 \%$ estaban casados y el $22 \%$ solteros.

Las labores administrativas eran las que más prevalecen (18\%), seguidas del trabajo en la construcción (9\%) y las tareas de limpieza (8\%). El 85 \% pertenecían al Régimen General y el $13 \%$ al de Autónomos

Los tumores más frecuentes fueron pulmón (14\%), mama (14\%), colon (9\%), linfoma (9\%) y astrocitoma (8\%). Si diferenciamos por sexo, en la mujer los más frecuentes fueron mama (36 \%) y pulmón (11 \%), y en el hombre pulmón (16 \%) y colon-recto (10 \%). En cuanto a la distribución por sexos (fig. 1) se observó que casi la totalidad de los tumores ORL y también la mayoría de los astrocitomas se presentaban en varones. Si diferenciamos por edades la frecuencia de cáncer de mama, pulmón y colon aumentan con la edad. No se encontró en la muestra ningún patrón que asocie profesión con tipo de cáncer, salvo la relación entre la profesión de comercial y las neoplasias relacionados con el tabaco (el 60 \% de sus cánceres eran de laringe o pulmón). 
Tabla II. Características de la muestra, de los fallecidos antes de los 5 años de seguimiento y de los supervivientes tras 5 años de seguimiento

\begin{tabular}{|c|c|c|c|c|c|c|c|c|c|}
\hline & & \multicolumn{2}{|c|}{$\begin{array}{l}\text { Características } \\
\text { de la muestra }\end{array}$} & \multicolumn{3}{|c|}{$\begin{array}{c}\text { Características de los } \\
\text { fallecidos antes } \\
\text { de los } 5 \text { años de seguimiento }\end{array}$} & \multicolumn{3}{|c|}{$\begin{array}{c}\text { Características } \\
\text { de los supervivientes } \\
\text { tras } 5 \text { años de seguimiento }\end{array}$} \\
\hline $\mathbf{N}^{\circ}$ de pacientes & \multicolumn{3}{|c|}{212} & \multicolumn{3}{|c|}{148} & \multicolumn{3}{|c|}{64} \\
\hline $\begin{array}{l}\text { Edad, años } \\
\text { (media +/- DE) }\end{array}$ & \multicolumn{3}{|c|}{$46,9+/-7$} & \multicolumn{3}{|c|}{$47,3+/-6$} & \multicolumn{3}{|c|}{$45,8+/-8$} \\
\hline Sexo masculino & \multicolumn{3}{|c|}{131} & \multicolumn{3}{|c|}{92} & \multicolumn{3}{|c|}{39} \\
\hline Casados & \multicolumn{3}{|c|}{145} & \multicolumn{3}{|c|}{106} & \multicolumn{3}{|c|}{39} \\
\hline $\begin{array}{l}\text { Tipos de cáncer } \\
\text { más frecuentes (\%): }\end{array}$ & total & mujeres & varones & total & mujeres & varones & total & mujeres & varones \\
\hline Pulmón & 30 & 9 & 21 & 27 & 8 & 19 & 3 & 1 & 2 \\
\hline Mama & 29 & 29 & 0 & 24 & 24 & 0 & 5 & 5 & 0 \\
\hline Colon - recto & 20 & 7 & 13 & 12 & 5 & 7 & 8 & 2 & 6 \\
\hline Linfoma & 19 & 7 & 12 & 11 & 3 & 8 & 8 & 4 & 4 \\
\hline Astrocitomas & 17 & 1 & 16 & 8 & 1 & 7 & 9 & 0 & 9 \\
\hline Boca/lengua & 12 & 1 & 11 & 6 & 0 & 6 & 6 & 1 & 5 \\
\hline Estomago & 11 & 3 & 8 & 11 & 3 & 8 & 0 & 0 & 0 \\
\hline Laringe & 9 & 1 & 8 & 6 & 1 & 5 & 3 & 0 & 3 \\
\hline Faringe & 7 & 0 & 7 & 6 & 0 & 6 & 1 & 0 & 1 \\
\hline Leucemia & 6 & 3 & 3 & 3 & 1 & 2 & 3 & 2 & 1 \\
\hline Meningioma & 5 & 2 & 3 & 2 & 0 & 2 & 3 & 2 & 1 \\
\hline Páncreas & 5 & 2 & 3 & 4 & 2 & 2 & 1 & 0 & 1 \\
\hline Otros & 58 & 19 & 29 & 31 & 9 & 28 & 17 & 10 & 7 \\
\hline $\begin{array}{l}\text { Presentaban o } \\
\text { habían presentado } \\
\text { metástasis/recidiva }\end{array}$ & & 117 & & & 94 & & & 23 & \\
\hline $\begin{array}{l}\text { Patología asociada no } \\
\text { constitutiva de IPA* }\end{array}$ & & 26 & & & 20 & & & 6 & \\
\hline $\begin{array}{l}\text { Secuelas u otras } \\
\text { limitaciones } \\
\text { constitutivas de IPA }\end{array}$ & & 32 & & & 19 & & & 13 & \\
\hline Profesión & & & & & & & & & \\
\hline $\begin{array}{l}\text { Labores } \\
\text { administrativas }\end{array}$ & & 39 & & & 29 & & & 10 & \\
\hline $\begin{array}{l}\text { Trabajo en la } \\
\text { construcción }\end{array}$ & & 20 & & & 12 & & & 8 & \\
\hline Limpieza & & 18 & & & 12 & & & 6 & \\
\hline $\begin{array}{l}\text { Tareas de venta al } \\
\text { público }\end{array}$ & & 14 & & & 12 & & & 2 & \\
\hline Labores sanitarias & & 12 & & & 7 & & & 5 & \\
\hline Hostelería & & 10 & & & 8 & & & 2 & \\
\hline Otras*** & & 99 & & & 68 & & & 31 & \\
\hline $\begin{array}{l}\text { Régimen de afiliación } \\
\text { a la Seguridad Social }\end{array}$ & & & & & & & & & \\
\hline Régimen General & & 180 & & & 125 & & & 55 & \\
\hline Autónomos & & 28 & & & 21 & & & 7 & \\
\hline Empleadas del hogar & & 4 & & & 2 & & & 2 & \\
\hline
\end{tabular}




\section{Esquema del estudio}

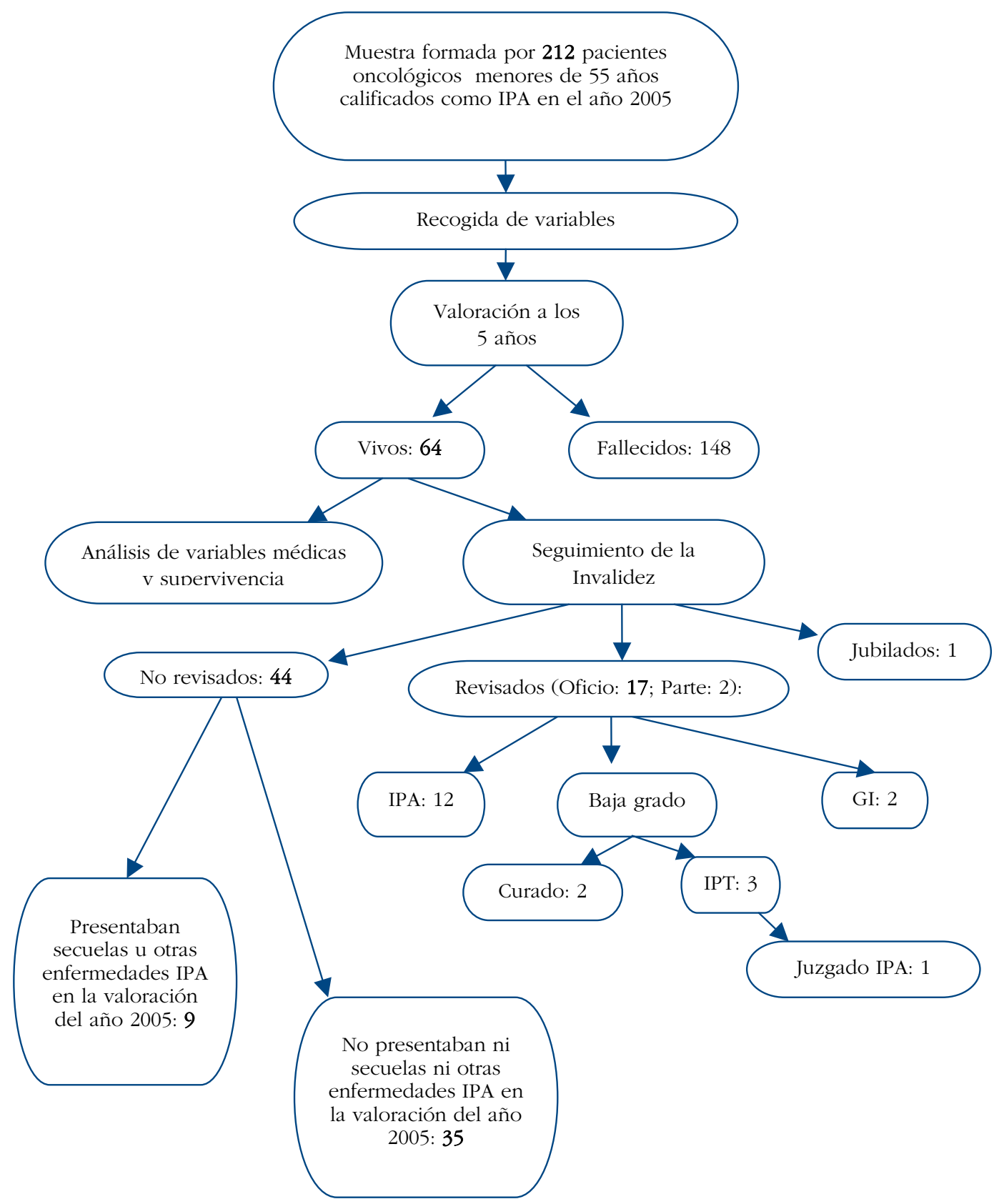


Figura 1. Distribución por sexos

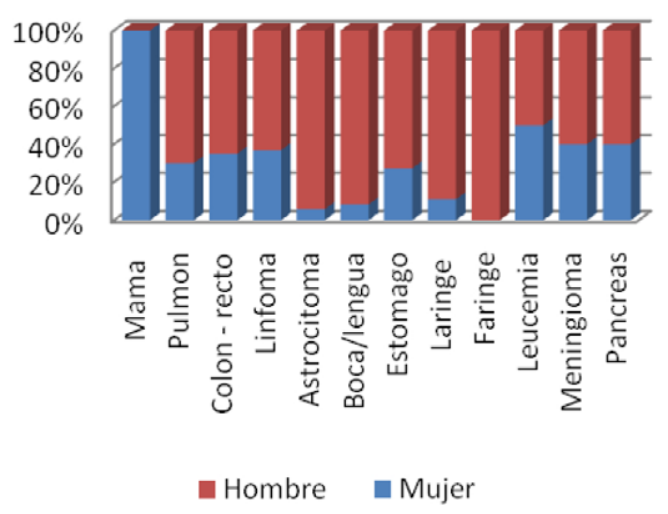

\section{Supervivencia}

El $30 \%$ (64 de los 212) de los pacientes a los que se les concedió una IPA seguían vivos al cabo de 5 años de la calificación de invalidez (más de 6 años desde el diagnóstico). El porcentaje de varones/mujeres fue similar al de la muestra inicial. Se apreciaron diferentes comportamientos respecto a la supervivencia global con las variables tipo de tumor (los peores datos los obtenemos con los tumores de estomago, pulmón y faringe) (fig. 2), edad (tendencia a disminuir la supervivencia a medida que aumenta la edad) (fig. 3), la presencia de metástasis y/o recidivas (reduce a menos de la mitad las posibilidades de supervivencia a 5 años) (fig. 4) y la presencia de patología asociada no constitutiva de IPA (fig.5), pero no con otras variables como el sexo. Los tumores que más muertes causan son pulmón en hombres y mama en mujeres. El tamaño de la muestra de supervivientes no permitió apreciar diferencias relevantes cuando asociamos las variables edad o sexo con cada tipo de tumor.

\section{Figura 2. Supervivencia a 5 años por tumores}

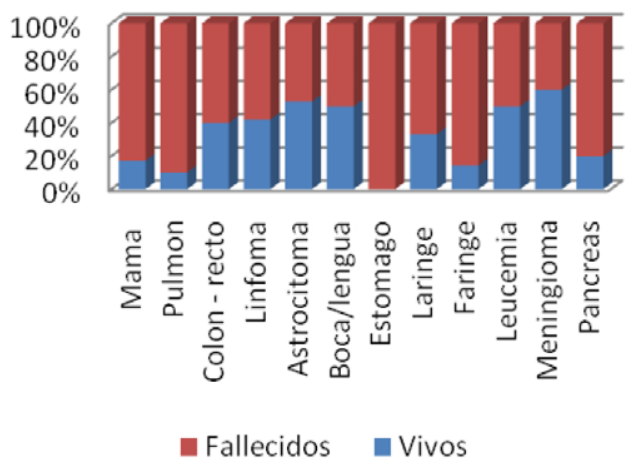

Figura 3. Supervivencia a 5 años en función de la edad

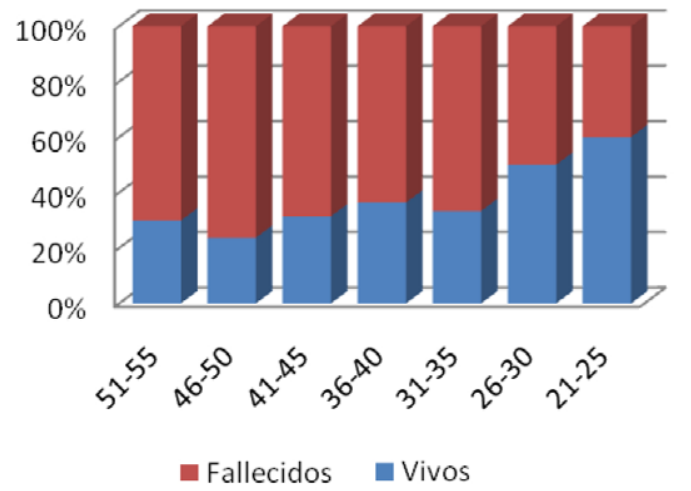

Paciente oncológico con incapacidad laboral absoluta: características epidemiológicas, 


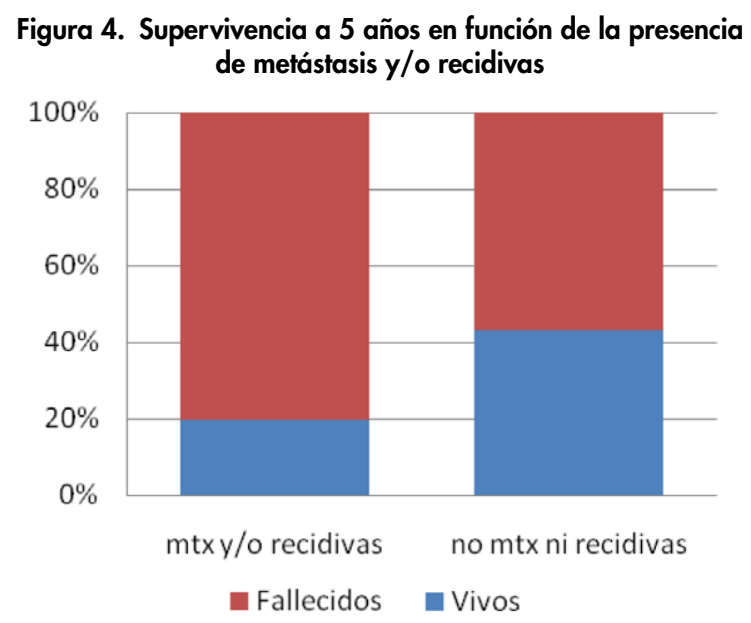

Figura 5. Supervivencia a 5 años en función de la presencia de patología asociada no constitutiva de IPA

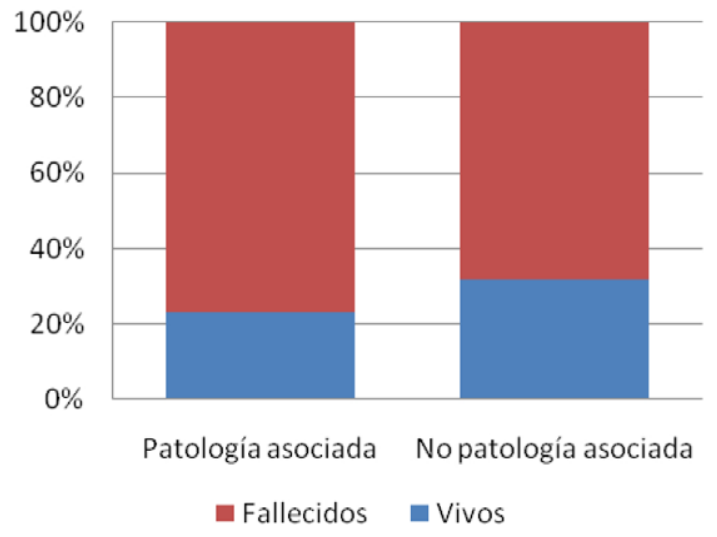

\section{Seguimiento de la Invalidez}

En cuanto a la concesión de invalidez, de los 64 pacientes que seguían vivos a los 5 años, 19 fueron revisados durante ese periodo. De los 44 no revisados, 35 no tenían secuelas ni otras patologías constitutivas IPA (16\% de la muestra), es decir, se les concedió el grado absoluto de invalidez por el estado físico que presentaban en el momento de la valoración y por el probable pronóstico de su enfermedad. En cuanto a la recuperación de la capacidad laboral, los 2 pacientes que volvieron a su trabajo (delineante y albañil) padecían linfoma Hodgkin, coincidiendo con el buen pronóstico que actualmente tienen estos tumores.

\section{DISCUSIÓN}

La literatura médica refleja ${ }^{11}$, que en mujeres el cáncer con mayor incidencia es el de mama y en hombres el de pulmón. Si hablamos conjuntamente de mujeres y hombres el cáncer colorectal es el más frecuente, pero no en nuestra muestra, por tratarse de un tumor que aparece sobre todo a partir de los 50 años (como ocurre con el de próstata), y las edades de nuestra muestra se sitúan entre 22-55 años. Los resultados también confirman el hecho de que los tumores ORL son significativamente más frecuentes entre varones.

Pese a que solo incorporamos población laboral, los varones representan el $62 \%$ de la muestra, porcentaje similar al de la población oncológica general. 
Es llamativo el elevado número de casos de astrocitoma. Su elevada supervivencia se debe a que en 6 casos se trataba de astrocitomas de bajo grado.

Desde el punto de vista laboral se observa que casi $2 / 3$ (el $63 \%$ ) de los pacientes a los que concedemos una IPA tienen metástasis-recidivas y/u otras lesiones constitutivas de IPA.

Por otro lado, el porcentaje de afiliados al Régimen General de la Seguridad Social es el mismo que el que ha presentado la Comunidad de Madrid durante el año 2010. A diferencia de otras patologías, el Régimen de Autónomos y el Régimen General muestran un comportamiento similar en cuanto a la incapacidad laboral.

\section{Supervivencia}

Se valoró el estado de los pacientes en noviembre del 2010, es decir, al menos 5 años desde la fecha de la resolución de invalidez

La $\mathrm{SEOM}^{2}$ publicó en enero del 2009 los siguientes datos de supervivencia a 5 años para el cáncer en España: Global 50,3 \%; Páncreas 5,3 \%; Pulmón 10,7 \%; Hígado 11,5 \%; SNC 17,1 \%; Estomago 27,8 \%; Mieloma Múltiple $34 \%$; Ovario 36,9 \%; Cabeza y cuello 39,4 \%; Leucemias $44 \%$; LNH 51,9 \%; Colorectal 53,6 \%; Riñón 58,9 \%; Cérvix 62,7 \%; Útero 73,1 \%; Vejiga 73,7 \%; E. Hodking 80,1\%; Mama 80,3\%; Tiroides $82 \%$; Melanoma $83,7 \%$; Testículo 94,9\%. Nosotros vamos a tomar como referencia los datos publicados recientemente por Chirlaque et $\mathrm{al}^{10}$, también referidos a la población española. Dicho trabajo forma parte del proyecto europeo Eurocare y recoge datos de la supervivencia de 9 tipos de tumores (colon, recto, pulmón, mama, próstata, ovario, testículo, melanoma de piel y linfoma Hodgkin) diagnosticados en el margen temporal 1995-1999 con una muestra representativa de toda la población española (cubre aproximadamente un $16 \%$ de la población comprendiendo 10 provincias: Álava, Guipúzcoa, Vizcaya, Navarra, Gerona, Tarragona, Castellón, Albacete, Murcia y Granada). Al ser la nuestra una población de pacientes oncológicos con mala evolución, al comparar dichos resultados con los nuestros, tanto si diferenciamos por sexo (fig. 6) como por tipo de tumor (fig. 7), obtenemos peores datos de supervivencia.

Las diferentes resultados que muestran hombres y mujeres puede deberse a que los tumores femeninos (mama, endometrio,...) son más curables que los más frecuentes en el varón (pulmón, colorectal,...). En nuestra muestra esta diferencia es menor debido a tener un escaso número de tumores ginecológicos (si excluimos el de mama).

Figura 6. Diferencia de supervivencias globales por sexo

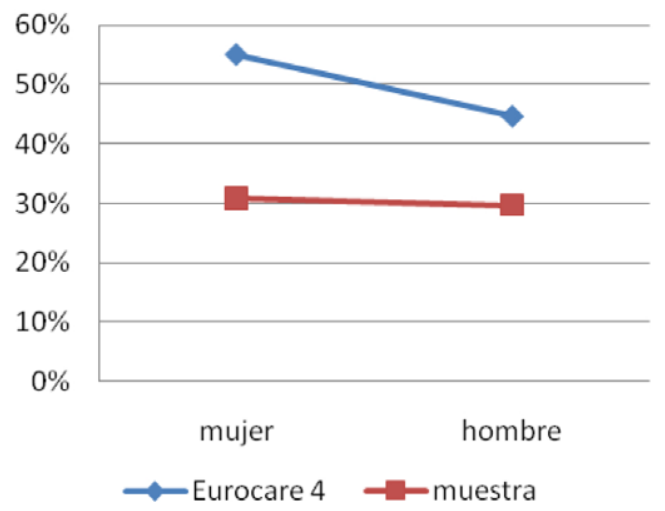

Los malos resultados del cáncer de mama se explica por el hecho de que a los 18 meses de IT solo llegan los estadios muy avanzados, de hecho, de las 29 neoplasias de 
mama de la muestra 24 presentaban metástasis. Por este motivo tampoco hemos obtenido peores resultados de supervivencia del cáncer de mama en mujeres jóvenes.

Al igual que en otros estudios ${ }^{11}$, observamos que los tumores que causan más muertes son el de pulmón en varones y el de mama en la mujer, y que la supervivencia global empeora con la edad, la presencia de patología asociada, la extensión tumoral (TNM) y la presencia de recidivas.

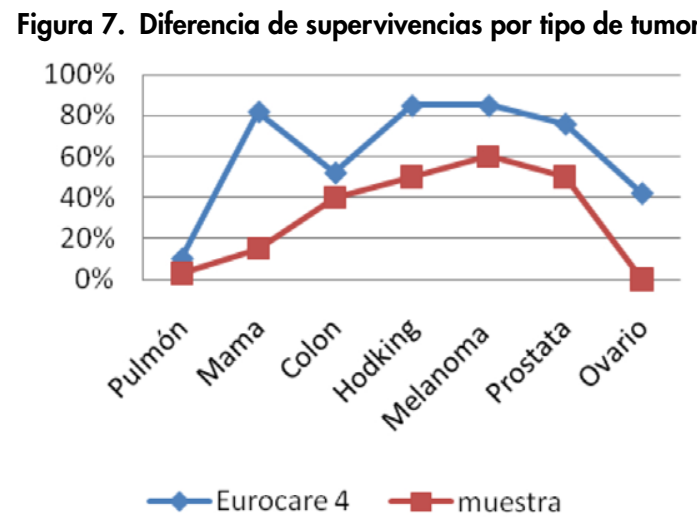

Los profesionales que evaluamos este tipo de pacientes debemos conocer el pronóstico de estas patologías. Para mejorar la precisión del pronóstico se pueden usar varios factores relacionados con la supervivencia. Estos factores podemos agruparlos:

- Factores dependientes del paciente, como la presencia de síntomas generales (síndrome constitucional, disnea, astenia,...), la edad, el órgano o sistema afectado, el estado general o performance status (nos lo aportará la aplicación de las escalas como las de Karnofsky y ECOG) y la presencia de patologías asociadas.

- Factores dependientes del tumor, como la localización (resecable o no resecable), la extensión (TNM), el grado de diferenciación histológica, índice proliferativo, estudio marcadores de membrana, análisis del contenido de ADN, marcadores tumorales (su presencia refleja la actividad de la neoplasia y tiene su principal utilidad en el seguimiento de la evolución de la misma), la presencia de número elevado de microvasos, o la sobrexpresión de oncogenes.

- Factores dependientes del tratamiento. Debemos diferenciar bien los conceptos de remisión completa o respuesta completa al tratamiento (desaparición de toda evidencia tumoral registrada en 2 mediciones consecutivas en el plazo mínimo de 1 mes; las remisiones completas se consideran el primer paso para la curación de las neoplasias), supervivencia (estar vivo a los 5 años de hacerse el diagnóstico) y curación del cáncer (entendemos que un tumor se ha curado cuando la curva de supervivencia del paciente es igual a la de de un grupo de población normal residente en la misma zona geográfica y con la misma clasificación por sexo, edad y grupo socioeconómico). En ciertos tipos de cáncer, como el cáncer de mama y el de próstata, muchos pacientes que sobreviven durante cinco años, con el tiempo acaban muriendo a causa de la enfermedad. Patricia Tai et al ${ }^{12}$ sugieren determinado umbral de años antes de estimar el tumor como estadísticamente curado, por ejemplo, 9 años para el cáncer de pulmón, 10`4 para el de ovario, $12^{\prime} 2$ para el de colon, 18`2 para el melanoma, 24`6 para el de próstata o 36`2 para el de mama.

Se han publicado otros factores pronósticos como los índices predictivos Palliative Prognostic Score (PaP) y Palliative Prognostic Index (PPI) ${ }^{13}$, índices de calidad de vida ${ }^{14}$ como el SF-36, escalas subjetivas como la estimación clinica de la supervivencia (ECS) ${ }^{15}$, datos de laboratorio como leucocitosis, linfopenia, niveles de proteína $\mathrm{C}$ reactiva ${ }^{16-18}$, etc., pero estos son de aplicación en el paciente oncológico terminal ${ }^{19}$. 


\section{Seguimiento de la Invalidez}

Por un lado tenemos que solo el $32 \%$ de los pacientes revisables (descartados los que presentaban secuelas u otras patologías constitutivas de IPA, el jubilado y las revisiones de parte) fueron revisados de oficio y que el $16 \%$ de los pacientes de la muestra permanecen vivos, sin secuelas u otras patologías constitutivas de IPA y sin revisiones previas.

Por otro lado tenemos que:

- Aumenta la incidencia del cáncer. El proyecto GLOBOCAN 2002, de la International Agency for Research on Cancer (IARC) ${ }^{20}$ calcula que la incidencia global prevista de cáncer para la población española en el año 2015 es de 222.069 personas (136.961 varones y 85.108 mujeres), siendo el tipo más frecuente el cáncer colorectal, por delante, en términos globales, del cáncer de pulmón y el cáncer de mama.

- Aumenta la supervivencia y la curación del cáncer. La SEOM ${ }^{2}$ recoge la evolución de la supervivencia en España comparando los datos del Eurocare 3 (pacientes diagnosticados en 1990-1994) y los del Eurocare 4 (pacientes diagnosticados en 2000-2002). Se observa que la supervivencia global en España pasa del $44 \%$ al $49,5 \%$ en varones y del 56,4 al $59 \%$ en mujeres.

- El elevado y creciente gasto económico que suponen las IPA por procesos neoplásicos. En cuanto al número total de IPA concedidas, según datos del Ministerio de Trabajo ${ }^{21}$ a 1 de Septiembre del 2010, en la Dirección Provincial de Madrid el 42,1 \% (30.916) de las pensiones por incapacidad médica concedidas este año son por IPA, con un saldo medio de 1.177 euros. Según dicha fuente Madrid es la 4. ${ }^{a}$ Comunidad Autónoma en número de concesiones de IPA por detrás de Andalucía, Cataluña y Valencia, y la 2. ${ }^{a}$ provincia después de Barcelona.

\section{CONCLUSIONES}

La medicina evaluadora debe adaptarse a estos nuevos cambios y puede hacerlo actualizando su formación oncológica y aumentando las revisiones de estos pacientes. En las revisiones podemos actuar a 2 niveles:

En el momento de decidir si la calificación de IPA debe ser revisada. Hemos visto que aunque la supervivencia de los tumores de nuestros pacientes es más baja que los de la población general, su relación con otras variables es similar y por tanto debemos aplicar a cada tumor sus factores pronósticos con el objetivo de establecer la necesidad de revisión de sus IPA. Los resultados de un trabajo realizado a nivel nacional en el Instituto Nacional de la Seguridad Social (INSS) en el año 2008 nos muestran que el capítulo de las neoplasias supone el $3 .^{\circ}$ lugar en frecuencia de las revisiones de oficio (tras la patología osteomioarticular y los trastornos mentales) y el $1 .^{\circ}$ en porcentaje de disminuciones del grado de invalidez (27,3\%), es decir, se realizan un importante número de revisiones y en un elevado porcentaje se objetiva mejoría. La pregunta es ¿si se indicasen más revisiones el porcentaje de disminuciones de grado sería mayor? Los médicos evaluadores debemos indicar la revisión de oficio siempre que tras consultar la información médica necesaria mantengamos dudas sobre la posibilidad de una mejoría suficiente para disminuir el grado de invalidez.

En los departamentos de invalidez de las distintas Direcciones Provinciales del INSS. Seleccionando, para ser revisados de oficio, aquellos casos que con una supervivencia, por ejemplo, mayor a 5 años y una edad razonable para reincorporarse al mundo laboral, no presenten otras patologías o secuelas constitutivas de IPA. Aunque sería recomendable la realización de un estudio piloto, considero que tras una selección adecuada de los expedientes, estas "revisiones a posteriori" probablemente permita adecuar el grado de incapacidad laboral a un porcentaje significativo de casos. 


\section{LIMITACIONES DEL ESTUDIO}

Se trata de un estudio exploratorio con un número bajo de pacientes para poder realizar un análisis multivariante. Por otro lado solo se han recogido algunas de las variables que se relacionan con el pronóstico (sexo, edad, extensión, comorbilidad y tipo de tumor), no pudiéndose relacionar con otros factores pronósticos recogidos en la literatura médica (performance status, grado de diferenciación, histología,...).

No obstante, dada la escasa cantidad de estudios sobre la reincorporación laboral de los pacientes oncológicos, nuestros resultados son interesantes para futuros proyectos que analicen las repercusiones económicas de la creciente prevalencia de estas patologías.

\section{AGRADECIMIENTOS}

A la Dirección Provincial del INSS de Madrid y a la Subdirección General de Coordinación de Unidades Médicas del INSS sin cuya colaboración y apoyo no hubiera sido posible la realización de este trabajo.

\section{REFERENCIAS BIBLIOGRÁFICAS}

1. Cabanes Domenech A, Pérez-Gómez B, AragonésN, Pollán M, López-Abente G. La situación del cáncer en España, 1975-2006. Madrid: Instituto de Salud Carlos III, 2009. http://cne.isciii.es

2. http://www.seom.org/prensa/el-cancer-en-espanyacom

3. Berrino F et al. and the EUROCARE working group (2007) Survival for eight major cancers and all cancers combined for European adults diagnosed in 1995-1999: results of the EUROCARE-4 study. Lancet Oncol 8: 773-783.

4. López Bastida J, Serrano Aguilar P, Duque González B. Los coste socioeconómicos de las enfermedades cardiovasculares y del cáncer en las Islas Canarias en 1998. Gaceta Sanitaria 2003; 17. 34.

5. Aaronson NK. Methodologic issues in assessing the quality of life of cancer patients. Cancer 1991;67:844-50.

6. Oliva J. Pérdidas de producción laboral ocasionadas por los fallecimientos prematuros en España en el año 2005. Rev Esp Salud Pública 2009; 83: 123-135.

7. Hartunian NS, Smart ChN, Thompson MS. The incidence and economic costs of cancer, motor vehicle injuries, coronary heart disease and stroke: a comparative analysis. AM J Public Health 1980; 70: 1249-60.

8. Kiiskinen U, Vartiainen E, Pekurinen M, Puska P.Does prevention of cardiovascular diseases lead to decreased cost of illness? Twenty years of experience from Finland. Prev Med 1997; 26: 220-6.

9. Chan B, Coyte P, Heick C. Economic impact of cardiovascular disease in Canada. Can J Cardiol 1996; 10: $255-9$.

10. Real decreto $1 / 1994$ de 20 de Junio, por el que se aprueba el Texto refundido de la Ley General de la seguridad Social.

11. Chirlaque M. D. et al Cancer survival in Spain: estimate for nine major cancers, Annals of Oncology 21 (Supplement 3): iii21-iii29, 2010.

12. Tai $\mathbf{P}$ et al Minimum follow-up time required for the estimation of statistical cure of cancer patients verification using data from 42 cancer sites in the SEER database. BMC Cancer 2005, 5:48; http://www. biomedcentral.com/1471-2407/5/48.

13. Maltoni M, Caraceni A, Brunelli C, Broeckaert B, Christakis N, Eychmueller S, et al. Prognostic factors in advanced cancer patients: Evidence-based clinical recommendations - A study by the Steering Committee of the European Association for alliative Care. J Clin Oncol. 2005;23:6240-8.

14. Glare P, Christakis N. Predicting survival in patients with advanced disease. En: Doyle D, Hanks G, Cherny N, Calman K, editors. Oxford Textbook of Palliative Medicine. Oxford: Oxford University Press; 2004. p. 29-42.

15. De Arriba Méndez JJ. Pronóstico de supervivencia en el cáncer avanzado. Rev Clin Esp. 2007;207(7):348-51.

16. Pirovano M, Maltoni M, Nanni O, Marinari M, Indelli M, Zaninetta G, et al. A new palliative prognostic score: a first step for the staging of terminally ill cancer patients. Italian multicenter and study group on palliative care. J Pain Symptom Manage. 1999;17:231-9. 
17. Maltoni M, Pirovano M, Nanni O. Biological indices predictive of survival in 591 italian terminally ill cancer patients. J Pain Symptom Manage. 1997; 13:1-9.

18. Geissbubler P, Mermilled B, Rain CH. Elevated serum vitamin B12 levels associated with CRP as a predictive factor of mortality in palliative care cancer patients: a prospective study over five years. J Pain Symptom Manage. 2000; 20:93-103.

19. De Miguel Sánchez C et al. El paciente con cáncer avanzado. ¿Podemos predecir la supervivencia? Aten Primaria. 2006; 38(Supl 2):2-6.

20. Ferlay J, Bray F, Pisan P, Parkin DM. GLOBOCAN 2002: Cancer Incidente, Mortality and Prevalence Worldwide. IARC CancerBase n. ${ }^{\circ}$ 5, version 2.0 Lyon IARC Press 2004. http://www-dep.iarc.fr2.

21. http://www.seg-social.es/Internet_1/Estadistica/Est/index.htm.

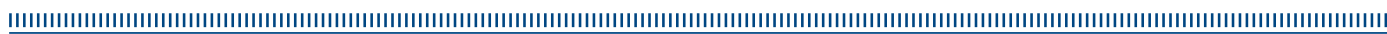

